\title{
Activity of any neuron with delayed feedback stimulated with Poisson stream is non-Markov
}

\author{
Alexander K.Vidybida *
}

\begin{abstract}
For a class of excitatory spiking neuron models with delayed feedback fed with a Poisson stochastic process, it is proven that the stream of output interspike intervals cannot be presented as a Markov process of any order.

Keywords. spiking neuron; Poisson stochastic process; probability density function; delayed feedback; non-Markov stochastic process
\end{abstract}

\section{Introduction}

Statistics of neuronal activity is often described as a renewal point process, or even a Poisson process, see [1] and references therein. On the other hand, in some sets of experimental data correlations are observed between consecutive interspike intervals (ISI), [2, 3, 4, 5], which does not conform with the renewal hypothesis. What could be the reason of such correlations? In principle, any sort of memory in the neuronal firing mechanism could bring about memory into the sequence of ISIs, thus disrupting a possibility for it to be renewal. Memory in the firing mechanism can appear due to partial reset of the membrane potential after firing, [6, 7], or due to threshold fatigue [8], or for other reasons, see [9] for a review.

Biologically, non-renewal statistics of neuronal activity can improve discrimination of weak signals [3, 9] and therefore is essential feature of functioning of a nervous system. In this context, it was checked in [3] if it is possible to represent activity of electrosensory neuron as a Markov chain of some finite order. Conclusion made in [3] is that the corresponding order, if any, cannot be lower than 7 .

\footnotetext{
*Bogolyubov Institute for Theoretical Physics, Metrologichna str., 14-B, Kyiv 03680, Ukraine, vidybida@bitp.kiev.ua, http://www.bitp.kiev.ua/pers/vidybida
} 
Normally, any neuron is embedded into a network. Inter-neuronal communication in the network is delayed due to finite speed of nervous impulses. In a reverberating network, this brings about one more reason for non-renewal firing statistics - the delayed feedback. We study here the simplest possible case of a network - a single neuron with delayed feedback. In the previous paper [10], it was proven for a concrete neuronal model - the binding neuron with threshold 2 - stimulated with Poisson stream of input impulses, that statistics of its ISIs is essentially non-Markov. In this paper, we refine and extend methods of [10] making those applicable to any neuron, which satisfies a number of very simple and natural conditions (see Cond0-Cond4 in $\mathrm{n}$. 2.1). Under those conditions, we prove rigorously that ISI statistics of a neuron with delayed feedback cannot be represented as a Markov chain of any finite order.

\section{Definitions and assumptions}

\subsection{Neuron without feedback}

We do not specify any concrete neuronal model, only expect that a neuron satisfies the following conditions:

- Cond0: Neuron is deterministic: Identical stimuli elicit identical spike trains from the same neuron.

- Cond1: Neuron is stimulated with input Poisson stream of excitatory impulses. The input stream has intensity $\lambda$.

- Cond2: Neuron may fire a spike only at a moment when it receives an input impulse.

- Cond3: Just after firing, neuron appears in its standard state, which is always the same.

- Cond4: The output interspike interval (ISI) distribution is characterized with a probability density function (pdf) $p^{0}(t)$, which is positive: $t>0 \Rightarrow p^{0}(t)>0$, and bounded: $\sup _{t>0} p^{0}(t)<\infty$.

The Cond0, above, is imposed in accordance with experimental observations, see e.g. [11, 12]. As regards the Cond1, Poisson stream is a standard stimulation when neuronal random activity is studied. The Cond2, above, is satisfied for most threshold-type neuronal models, starting from standard leaky integrate and fire (LIF) neuron [13] and its modifications, see [14]. In 
order the Cond2 to be valid, it is enough that the following three conditions are satisfied: (i) neuronal excitation ${ }^{1}$ gets abrupt increase at the moment of receiving input impulse ${ }^{2}$, (ii) after that moment, the degree of excitation does not increase (it decreases for most neuronal models) until the next input impulse. (iii) the neuron fires when its degree of excitation exceeds a threshold level. The threshold can be either static, as in the basic LIF model, or dynamic [15]. These conditions seem to be standard for many threshold neuronal models used, see [8, 16, 17] and citations therein. Cond3 means that any kind of memory about previous input/output activity, which can be present in a neuron, is cleared after each triggering. Due to Cond3, output stream of neuron without feedback will be a renewal stochastic process. Cond4 seems to be natural for any neuronal model stimulated with Poisson stream. At least, all the five conditions are satisfied for the binding neuron model and for the basic LIF model, see [18, 19], where $p^{0}(t)$ is calculated exactly for each model, respectively.

\section{$2.2 \quad$ Feedback line action}

We expect that each output impulse fired by neuron is fed back to the neuron's input through a feedback line. The feedback line has the following properties:

- Prop1: The time delay in the line is $\Delta>0$.

- Prop2: The line is able to convey no more than one impulse.

- Prop3: The impulse conveyed to the neuronal input is identical to that from the input Poisson stream.

It is known that a neuron can form synapses (autapses) on its own body, or dendritic tree, e.g. [20, 21]. This substantiates consideration of a single neuron with feedback not only as the simplest reverberating "network" possible, bat also as an independent biologically relevant case. The delay $\Delta$ comprises the time required by the output spike to pass the distance from axonal hillock, where it is generated, to the autapse and the synaptic delay. The Prop2 is somehow related to the refractoriness even if we do not introduce here the refractoriness to its full extent. The Prop3 means that we consider here an excitatory neuron.

\footnotetext{
${ }^{1}$ We use here term "excitation" instead of "depolarization voltage" because we do not specify any triggering mechanism. Our consideration as regards feedback shaping of firing statistics could be valid also for essentially artificial neurons, where excitation not necessarily has a voltaic nature.

${ }^{2}$ If considering an input impulse as a current impulse, then it has a $\delta$-function form.
} 
The important for us consequence of Prop2 is that at any moment of time the feedback line is either empty, or conveys a single impulse. If it does convey an impulse, then its state can be described with a stochastic variable $s$, which we call further "time to live". The variable $s$ denotes the exact time required by the impulse to reach the output end of the line, which is the neuron's input, and to leave the line. It is clear that $0<s \leq \Delta$. In what follows, we use the time to live $s$ only at moments when an ISI starts (just after triggering).

Now it is worth to notice that each triggering starts a new ISI. And at the beginning of any ISI the line is never empty, but holds an impulse. This happens for the following reasons:

a) If neuron is triggered by an impulse from the Poisson input stream, and the line was empty just before that moment, then the emitted impulse enters the line. At that moment the line is characterized with $s=\Delta$.

b) If neuron is triggered by an impulse from the Poisson input stream, and the line already conveys an impulse at that moment with time to live $s$, then that same impulse with that same time to live is retained at the beginning of the ISI that starts after that triggering, and the line is characterized with that same $s$.

c) If neuron is triggered by an impulse from the line, then the line is empty at the firing moment and the emitted impulse enters the line. After that moment the line is characterized with $s=\Delta$.

\subsection{Proof outline}

We expect that defined in $\mathrm{nn}$. 2.1, 2.2 system of neuron with delayed feedback line fed with Poisson stream is in its stationary regime. This can be achieved if the system functions long enough that its initial state is forgotten.

In the stationary regime, let $p\left(t_{n}, \ldots, t_{1}\right)$ denotes the joint probability density function of neuron with delayed feedback. The probability to get, in the output, starting from the beginning, $n$ consecutive ISIs $t_{1}^{\prime}, \ldots, t_{n}^{\prime}$ such that $t_{i}^{\prime} \in\left[t_{i} ; t_{i}+d t_{i}\left[, i=1, \ldots, n\right.\right.$ with infinitesimal $d t_{i}$ is given by $p\left(t_{n}, \ldots, t_{1}\right) d t_{1} \ldots d t_{n}$.

Let $p\left(t_{n+1} \mid t_{n}, \ldots, t_{0}\right) d t_{n+1}$ denotes the conditional probability to get the duration of $(n+2)$-th ISI in $\left[t_{n+1} ; t_{n+1}+d t_{n+1}\right.$ [ provided that previous $n+1$ ISIs had duration $t_{n}, \ldots, t_{0}$, respectively.

Now we reformulate in terms of probability density functions the definition from [22, Ch.2 §6]: 
Definition 1. The sequence of random variables $\left\{t_{j}\right\}$, taking values in $\Omega$, is called the Markov chain of the order $n \geq 0$, if

$$
\forall_{m>n} \forall_{t_{0} \in \Omega} \ldots \forall_{t_{m} \in \Omega} p\left(t_{m} \mid t_{m-1}, \ldots, t_{0}\right)=p\left(t_{m} \mid t_{m-1}, \ldots, t_{m-n}\right),
$$

and this equation does not hold for any $n^{\prime}<n$.

In particular, taking $m=n+1$, we have the necessary condition

$$
p\left(t_{n+1} \mid t_{n}, \ldots, t_{1}, t_{0}\right)=p\left(t_{n+1} \mid t_{n}, \ldots, t_{1}\right), \quad t_{i} \in \Omega, i=0, \ldots, n+1,
$$

required for the stochastic process $\left\{t_{j}\right\}$ to be $n$-order Markov chain. In the case of ISIs one reads $\Omega=\mathbb{R}^{+}$.

We intend to prove that the relation (1) does not hold for any $n$. For this purpose we calculate exact expression for $p\left(t_{n+1} \mid t_{n}, \ldots, t_{0}\right)$ as

$$
p\left(t_{n+1} \mid t_{n}, \ldots, t_{0}\right)=\frac{p\left(t_{n+1}, t_{n}, \ldots, t_{0}\right)}{p\left(t_{n}, \ldots, t_{0}\right)}
$$

from which it will be clearly seen that the $t_{0}$-dependence in $p\left(t_{n+1} \mid t_{n}, \ldots, t_{0}\right)$ cannot be eliminated whatever large the $n$ is.

As it is seen from (2), we need initially to calculate exact expressions for $p\left(t_{n}, \ldots, t_{0}\right)$ with arbitrary $n$. In [10, for the binding neuron model with threshold 2 this is done by introducing an auxiliary stochastic process with events $\left(t_{i}, s_{i}\right)$, where $s_{i}$ is the time to live at the beginning of ISI $t_{i}$. It was proven that the sequence of events $\left(t_{i}, s_{i}\right), i=0,1, \ldots$, is Markov chain, which helps to calculate the joint probability density $p\left(\left(t_{n}, s_{n}\right), \ldots,\left(t_{0}, s_{0}\right)\right)$ and then $p\left(t_{n}, \ldots, t_{0}\right)$ as marginal probability by integrating it over $\left.] 0 ; \Delta\right]$ with respect to each $s_{i}$. To simplify this approach, it is worth to notice that in the sequence of consecutive random events $\left(t_{n}, s_{n}\right), \ldots,\left(t_{0}, s_{0}\right)$ only the values of variables $t_{n}, \ldots, t_{1}, t_{0}, s_{0}$ are fairly random. Indeed, with $t_{0}, s_{0}$ given, one can figure out exact value for the $s_{1}$ : if $t_{0}<s_{0}$ then $s_{1}=s_{0}-t_{0}$, and $s_{1}=\Delta$ otherwise. Now, with $t_{1}, s_{1}$ known, the same way it is possible to find the exact value of $s_{2}$ and so on. This allows one to reconstruct unambiguously all the values $s_{1}, \ldots, s_{n}$ from the given sequence of values of $t_{n}, \ldots, t_{1}, t_{0}, s_{0}$. Having this in mind, we introduce the conditional joint probability density $p\left(t_{n+1}, \ldots, t_{0} \mid s\right)$, which we use to calculate required joint pdfs as follows

$$
p\left(t_{n+1}, \ldots, t_{0}\right)=\int_{0}^{\Delta} p\left(t_{n+1}, \ldots, t_{0} \mid s\right) f(s) d s
$$


where $s$ (dented previously as $s_{0}$ ) is the time to live at the beginning of ISI $t_{0}$, $f(s)$ is the stationary pdf which describes distribution of times to live at the beginning of any ISI in the stationary regime. In what follows we analyze the structure of functions $f(s)$ and $p\left(t_{n+1}, \ldots, t_{0} \mid s\right)$. It appears that $f(s)$ has a singular component $a \delta(s-\Delta)$ with $a>0$, and $p\left(t_{n+1}, \ldots, t_{0} \mid s\right)$ has a $\delta$ function-type singularities at definite hyper-planes in the $(n+2)$-dimensional space of its variables $\left(t_{n+1}, \ldots, t_{0}\right)$. After integration in (3), some of those $\delta$ functions will survive, and one of those survived has its argument depending on $t_{0}$. The latter statement depends on exact value of ISIs in the sequence $t_{n+1}, \ldots, t_{0}$. Here, we limit our consideration to the domain in the $(n+2)$ dimensional space of variables $\left(t_{n+1}, \ldots, t_{0}\right)$, which is defined as follows

$$
\sum_{i=0}^{n} t_{i}<\Delta
$$

Notice that $t_{n+1}$ is not involved in (4).

The $t_{0}$-dependent $\delta$-function will as well survive in the $p\left(t_{n+1} \mid t_{n}, \ldots, t_{0}\right)$ for any $n$, which will complete the proof that the condition (1) cannot be satisfied for any $n$.

A question remains of whether the domain (4) has a strictly positive probability. This indeed takes place due to positiveness of pdfs $p\left(t_{n+1}, \ldots, t_{0}\right)$ for any positive values of $\left(t_{n+1}, \ldots, t_{0}\right)$. The latter follows from the exact expressions for $p\left(t_{n+1}, \ldots, t_{0}\right)$ given in n. 3.4.1. Eq. (19).

\section{The proof}

\subsection{Structure of functions $p\left(t_{n+1}, \ldots, t_{0} \mid s\right)$}

Expect that the inequality (4) holds. In order to perform integration in (3), we split the integration domain into the following $n+2$ disjoint sub-domains:

$$
\left.\left.\left.\left.D_{k}=\right] \sum_{i=0}^{k-1} t_{i} ; \sum_{i=0}^{k} t_{i}\right], k=0, \ldots, n, \quad D_{n+1}=\right] \sum_{i=0}^{n} t_{i} ; \Delta\right] .
$$

It is clear that

$$
\left.\left.\bigcup_{k=0}^{n+1} D_{k}=\right] 0 ; \Delta\right]
$$

The conditional pdf $p\left(t_{n+1}, \ldots, t_{0} \mid s\right)$ has different structure at different domains. If $s \in D_{k}$, then a relation between $s$ and $t_{i}$ is as shown in Fig. 1 . As it could be suggested by Fig. 1, the first $k-1$ ISIs are produced with 


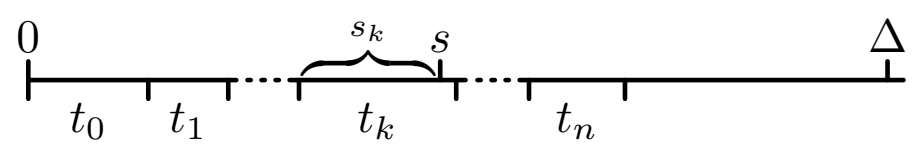

Fig 1: Mutual disposition in time of $s$ and $t_{0}, \ldots, t_{n}$ if $s \in D_{k}$.

the delay line not involved. The $k$-th ISI is generated with the line involved. The corresponding time to live is $s_{k}=s-\sum_{i=0}^{k-1} t_{i} \leq t_{k}$, the next time to live is $s_{k+1}=\Delta$. Therefore, the structure of $p\left(t_{n+1}, \ldots, t_{0} \mid s\right)$ at $D_{k}$ is as follows

$$
p\left(t_{n+1}, \ldots, t_{0} \mid s\right)=p\left(t_{n+1}, \ldots, t_{k+1} \mid \Delta\right) p\left(t_{k} \mid s-\sum_{i=0}^{k-1} t_{i}\right) \prod_{i=0}^{k-1} p^{0}\left(t_{i}\right)
$$

where $k=0,1, \ldots, n$. And if $s \in D_{n+1}$, then relation between $s$ and $t_{i}$ is as

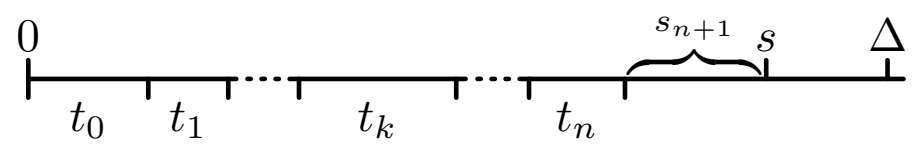

Fig 2: Mutual disposition in time of $s$ and $t_{0}, \ldots, t_{n}$ if $s \in D_{n+1}$.

shown in Fig. 22. This suggests the following structure for $p\left(t_{n+1}, \ldots, t_{0} \mid s\right)$

$$
p\left(t_{n+1}, \ldots, t_{0} \mid s\right)=p\left(t_{n+1} \mid s-\sum_{i=0}^{n} t_{i}\right) \prod_{i=0}^{n} p^{0}\left(t_{i}\right), s \in D_{n+1} .
$$

Here $p(t \mid s)$ denotes the conditional pdf to get ISI of duration $t$ if at its beginning, time to live of impulse in the feedback line is $s$.

By utilizing the same reasoning with (4) taken into account, one can represent the first factor in (5) as follows

$$
p\left(t_{n+1}, \ldots, t_{k+1} \mid \Delta\right)=p\left(t_{n+1} \mid \Delta-\sum_{i=k+1}^{n} t_{i}\right) \prod_{i=k+1}^{n} p^{0}\left(t_{i}\right)
$$

Representation of $p\left(t_{n+1}, \ldots, t_{0} \mid s\right)$ by means of $p^{0}(t)$ and $p(t \mid s)$, similar to that displayed in (5), (6), (7), can be as well constructed if (4) does not hold. For our purpose it is enough to have (5), (6) and (7). 


\subsection{Structure of function $p(t \mid s)$}

Expect that at the beginning of an ISI, there is an impulse in the feedback line with time to live $s$. Then the probability that this ISI will have its duration $t<s$ does not depend on the feedback line presence. Therefore,

$$
t<s \Rightarrow p(t \mid s)=p^{0}(t)
$$

The probability to get exactly $t=s$ is not zero, because in this case the impulse, which triggers the neuron and finishes the ISI under consideration comes from the delay line. In order this to happen, it is necessary and sufficient that the following two events take place: (i) the neuron does not fire at the interval $] 0 ; s[$; (ii) at the moment $s$, the neuron, due to previous stimulation from the Poisson stream, achieves such a state that adding one more input impulse will trigger it. The probability of (i) and (ii) is $\frac{p^{0}(s)}{\lambda}$, which can be easily concluded from the definition of $p^{0}(t)$. Thus,

$$
t \in] s-\epsilon ; s+\epsilon\left[\Rightarrow p(t \mid s)=\frac{p^{0}(t)}{\lambda} \delta(s-t)\right.
$$

with infinitesimal $\epsilon>0$. If the neuron still not triggered at moment $s$, then it is triggered by an input impulse from the Poisson stream at $t>s$. The probability to get such an impulse in $[t ; t+d t[$ is $\lambda d t$. Therefore, one can expect that for $t>s, p(t \mid s) \leq \lambda$.

Based on the above reasoning we represent $p(t \mid s)$ in the following form

$$
p(t \mid s)=p^{b}(t \mid s)+\frac{p^{0}(t)}{\lambda} \delta(s-t)
$$

where $p^{b}(t \mid s)$ is a bounded function $]^{3}$

\subsection{Structure of probability density function $f(s)$}

In the stationary regime, the pdf $f(s)$ must satisfy the following equation

$$
f(s)=\int_{0}^{\Delta} \mathbf{P}\left(s \mid s^{\prime}\right) f\left(s^{\prime}\right) d s^{\prime},
$$

where the transition function $\mathbf{P}\left(s \mid s^{\prime}\right)$ gives the probability density to find at the beginning of an ISI an impulse in the line with time to live $s$ provided

\footnotetext{
${ }^{3}$ Compare this with [23, Eq. (7)], where $p(t \mid s)$ is calculated exactly for the binding neuron model.
} 
at the beginning of the previous ISI, there was an impulse with time to live $s^{\prime}$.

To determine exact expression for $\mathbf{P}\left(s \mid s^{\prime}\right)$ we take into account that after single firing, time to live can either decrease, or become equal $\Delta$. Therefore,

$$
s^{\prime} \leq s<\Delta \Rightarrow \mathbf{P}\left(s \mid s^{\prime}\right)=0 .
$$

If $s<s^{\prime}$, then the firing, which causes transition from $s^{\prime}$ to $s$, happens without the line involved. Therefore,

$$
0<s<s^{\prime} \Rightarrow \mathbf{P}\left(s \mid s^{\prime}\right) d s=p^{0}\left(s^{\prime}-s\right) d s .
$$

Finally, it is possible that starting from $s^{\prime}$ one obtains $s=\Delta$ after the next firing. In order this to happen, it is necessary and sufficient that no firing happens during $s^{\prime}$ units of time. And this happens with probability

$$
\mathbf{P}^{0}\left(s^{\prime}\right)=1-\int_{0}^{s^{\prime}} p^{0}(t) d t
$$

Having this in mind, one could conclude that in the plane $\left(s, s^{\prime}\right)$, at the straight line $s=\Delta, s^{\prime}$ - any, the $\mathbf{P}\left(s \mid s^{\prime}\right)$ has singularity of the following form:

$$
\mathbf{P}^{0}\left(s^{\prime}\right) \delta(s-\Delta)
$$

Now, with (10)-(12) taken into account, Eq. (9) can be rewritten as follows

$$
f(s)=\int_{s}^{\Delta} p^{0}\left(s^{\prime}-s\right) f\left(s^{\prime}\right) d s^{\prime}+\delta(s-\Delta) \int_{0}^{\Delta} \mathbf{P}^{0}\left(s^{\prime}\right) f\left(s^{\prime}\right) d s^{\prime} .
$$

It is clear from this equation that $f(s)$ has the following form ${ }^{4}$

$$
f(s)=g(s)+a \delta(s-\Delta),
$$

where $a>0$ and $g(s)$ is bounded and vanishes out of interval $] 0 ; \Delta]$.

\footnotetext{
${ }^{4}$ Compare this with [23, Eqs. (14)-(16)], where $f(s)$ is calculated exactly for the binding neuron model.
} 


\subsection{Form of $p\left(t_{n+1}, \ldots, t_{0}\right)$ and $p\left(t_{n}, \ldots, t_{0}\right)$ after integra- tion in (3)}

Let $D=\bigcup_{k=0}^{n} D_{k}$. At $D$, representations 55 and 77 are valid. Also at $D, f(s)$ reduces to $g(s)$. Therefore,

$$
\begin{aligned}
& \int_{D} p\left(t_{n+1}, \ldots, t_{0} \mid s\right) f(s)= \\
& =\sum_{k=0}^{n} p\left(t_{n+1} \mid \Delta-\sum_{i=k+1}^{n} t_{i}\right) \prod_{\substack{i=0 \\
i \neq k}}^{n} p^{0}\left(t_{i}\right) \int_{D_{k}} p\left(t_{k} \mid s-\sum_{i=0}^{k-1} t_{i}\right) g(s) d s .
\end{aligned}
$$

Taking into account (8) it can be concluded that expression (14), after performing integration, does not have any term with $\delta$-function depending on $t_{0}$.

Consider now the remaining part of integral in (3). With (6) taken into account one has:

$$
\int_{D_{n+1}} p\left(t_{n+1}, \ldots, t_{0} \mid s\right) f(s)=\prod_{i=0}^{n} p^{0}\left(t_{i}\right) \int_{D_{n+1}} p\left(t_{n+1} \mid s-\sum_{i=0}^{n} t_{i}\right) f(s) d s .
$$

After substituting here expressions (8), (13) one obtains four terms:

$$
\begin{aligned}
\int_{D_{n+1}} p\left(t_{n+1}, \ldots,\right. & \left.t_{0} \mid s\right) f(s)= \\
= & \prod_{i=0}^{n} p^{0}\left(t_{i}\right) \int_{D_{n+1}} p\left(t_{n+1} \mid s-\sum_{i=0}^{n} t_{i}\right) f(s) d s \\
= & \prod_{i=0}^{n} p^{0}\left(t_{i}\right) \int_{D_{n+1}} p^{b}\left(t_{n+1} \mid s-\sum_{i=0}^{n} t_{i}\right) g(s) d s \\
& +a \prod_{i=0}^{n} p^{0}\left(t_{i}\right) p^{b}\left(t_{n+1} \mid \Delta-\sum_{i=0}^{n} t_{i}\right) \\
+ & \frac{1}{\lambda} \prod_{i=0}^{n+1} p^{0}\left(t_{i}\right) g\left(\sum_{i=0}^{n+1} t_{i}\right)+\frac{a}{\lambda} \prod_{i=0}^{n+1} p^{0}\left(t_{i}\right) \delta\left(\Delta-\sum_{i=0}^{n+1} t_{i}\right) .
\end{aligned}
$$

After performing integration, only the fourth term here includes a $\delta$-function. And argument of this $\delta$-function does depend on $t_{0}$. 
After taking (14) and (15) together we conclude that the required joint probability density has the following form

$$
p\left(t_{n+1}, \ldots, t_{0}\right)=p^{w}\left(t_{n+1}, \ldots, t_{0}\right)+\frac{a}{\lambda} \prod_{i=0}^{n+1} p^{0}\left(t_{i}\right) \delta\left(\Delta-\sum_{i=0}^{n+1} t_{i}\right),
$$

where function $p^{w}\left(t_{n+1}, \ldots, t_{0}\right)$ does not have singularities depending on $t_{0}$.

\subsubsection{Form of $p\left(t_{n}, \ldots, t_{0}\right)$ after integration}

If (4) is satisfied, then we have similarly to (5), (6)

$$
\begin{gathered}
p\left(t_{n}, \ldots, t_{0} \mid s\right)=p\left(t_{n}, \ldots, t_{k+1} \mid \Delta\right) p\left(t_{k} \mid s-\sum_{i=0}^{k-1} t_{i}\right) \prod_{i=0}^{k-1} p^{0}\left(t_{i}\right), \\
s \in D_{k}, \quad k=0, \ldots, n-1, \\
p\left(t_{n}, \ldots, t_{0} \mid s\right)=p\left(t_{n} \mid s-\sum_{i=0}^{n-1} t_{i}\right) \prod_{i=0}^{n-1} p^{0}\left(t_{i}\right), \quad s \in D_{n} .
\end{gathered}
$$

Again due to (4), and in analogy with (7) we have instead of the last two equations the following one:

$$
p\left(t_{n}, \ldots, t_{0} \mid s\right)=p\left(t_{k} \mid s-\sum_{i=0}^{k-1} t_{i}\right) \prod_{\substack{i=0 \\ i \neq k}}^{n} p^{0}\left(t_{i}\right),
$$

It is clear that expression similar to (6) turns here into the following

$$
p\left(t_{n}, \ldots, t_{0} \mid s\right)=\prod_{i=0}^{n} p^{0}\left(t_{i}\right), \quad s \in D_{n+1} .
$$

Now, due to (17), 18) we have

$$
\begin{aligned}
p\left(t_{n}, \ldots, t_{0}\right) & =\int_{0}^{\Delta} p\left(t_{n}, \ldots, t_{0} \mid s\right) f(s) d s= \\
& =\sum_{\substack { k=0 \\
\begin{subarray}{c}{i \neq 0 \\
i \neq k{ k = 0 \\
\begin{subarray} { c } { i \neq 0 \\
i \neq k } }\end{subarray}}^{n} p^{0}\left(t_{i}\right) \int_{D_{k}} p\left(t_{k} \mid s-\sum_{i=0}^{k-1} t_{i}\right) g(s) d s+ \\
& +\prod_{i=0}^{n} p^{0}\left(t_{i}\right) \int_{D_{n+1}} f(s) d s .
\end{aligned}
$$




\section{$3.5 \quad t_{0}$-dependence cannot be eliminated in $p\left(t_{n+1} \mid t_{n}, \ldots, t_{0}\right)$}

Now, with representations 116$)$ for $p\left(t_{n+1}, \ldots, t_{0}\right)$ and $(19 p)$ for $p\left(t_{n}, \ldots, t_{0}\right)$ we can pose a question about the form of $p\left(t_{n+1} \mid t_{n}, \ldots, t_{0}\right)$. The latter can be found as defined in (2). First of all notice that due to (19) and Cond4, $p\left(t_{n}, \ldots, t_{0}\right)$ is strictly positive for positive ISIs. This allows us to use it as denominator in the definition (2). Second, it can be further concluded from (19) and Cond 4 , that $p\left(t_{n}, \ldots, t_{0}\right)$ is bounded, and therefore does not include any singularity of $\delta$-function type. The latter means that any singularity contained in the $p\left(t_{n+1}, \ldots, t_{0}\right)$ appears as well in the $p\left(t_{n+1} \mid t_{n}, \ldots, t_{0}\right)$. It follows from the above that the conditional pdf $p\left(t_{n+1} \mid t_{n}, \ldots, t_{0}\right)$ can be represented in the following form:

$$
p\left(t_{n+1} \mid t_{n}, \ldots, t_{0}\right)=p^{w}\left(t_{n+1} \mid t_{n}, \ldots, t_{0}\right)+Q\left(t_{n+1}, \ldots, t_{0}\right) \delta\left(\Delta-\sum_{i=0}^{n+1} t_{i}\right),
$$

where $p^{w}\left(t_{n+1} \mid t_{n}, \ldots, t_{0}\right)$ does not contain any $\delta$-function depending on $t_{0}$, and $Q\left(t_{n+1}, \ldots, t_{0}\right)$ is strictly positive bounded function:

$$
Q\left(t_{n+1}, \ldots, t_{0}\right)=\frac{a \prod_{i=0}^{n+1} p^{0}\left(t_{i}\right)}{\lambda p\left(t_{n}, \ldots, t_{0}\right)}
$$

The representation (20) thus proves unequivocally that for any $n$, conditional pdf $p\left(t_{n+1} \mid t_{n}, \ldots, t_{0}\right)$ does depend on $t_{0}$ (the second term in (20) ) and this dependence cannot be eliminated.

\section{Conclusions and Discussion}

We have proven here that any neuronal model, which satisfies Cond0-Cond4, above, and is equipped with a delayed feedback, will display essentially nonMarkov activity expressed in terms of output ISIs, when stimulated with Poisson stream. This has a consequence for admissible approaches while modeling activity of neuronal networks with stochastic behavior. Indeed, in a reverberating network, a delayed feedback mediated by other neurons is always present. Our result suggests that in this case, activity of individual neurons in the network should be essentially non-Markov. Another situation in networks with instantaneous interneuronal communication. In the case of no delay communications, the neuronal activity can well be Markov, or even Poisson, see example in [24]. 
We used here a single neuron with delayed feedback as the simplest case of reverberating "network". At the same time, neurons which send to themselves their output impulses are known in real nervous systems, [20, 21. Therefore, our conclusions about essentially non-Markov behavior should be valid for those neurons even without taking into account their involvement in a wider network activity.

The set of conditions Cond0-Cond 4 while being rather natural and wide enough, leaves out of our consideration many neuronal models known in neuroscience. E.g., Cond2 excludes models with spike latency. Cond3 excludes models with internal memory extending beyond a single ISI duration. Thus, we do not consider here partial afterspike resetting [6, 7], threshold fatigue [8], another types of adaptation, like multi-timescale adaptive threshold [25]. Any kind of adaptation in individual neuron is by itself able to bring about a kind of memory in the neuronal output stream. Therefore, considering neurons without adaptation we demonstrate here, that delayed feedback without additional memory-like mechanisms known for neurons makes neuronal output essentially non-Markov.

Another limitation is Cond 1 - we use a Poisson process as a stimulus. It seems that the proof given here can be extended to a wide class of renewal processes taken as stimuli. This will be checked in further work.

\section{References}

[1] B. B. Averbeck. Poisson or not poisson: Differences in spike train statistics between parietal cortical areas. Neuron, 62(3):310-311, 2009.

[2] S. B. Lowen and M. C. Teich. Auditory-nerve action potentials form a nonrenewal point process over short as well as long time scales. The Journal of the Acoustical Society of America, 92(2):803-806, 1992.

[3] R. Ratnam and M. E. Nelson. Nonrenewal statistics of electrosensory afferent spike trains: implications for the detection of weak sensory signals. The Journal of Neuroscience, 20:6672-6683, 2000.

[4] M. P. Nawrot, C. Boucsein, V. Rodriguez-Molina, A. Aertsen, S. Grün, and S. Rotter. Serial interval statistics of spontaneous activity in cortical neurons in vivo and in vitro. Neurocomputing, 70:1717-1722, 2007.

[5] G. Maimon and J. A. Assad. Beyond poisson: Increased spike-time regularity across primate parietal cortex. Neuron, 62(3):426-440, 2009. 
[6] J. P. Rospars and P. Lansky. Stochastic model neuron without resetting of dendritic potential: application to the olfactory system. Biol. Cybern., 69(4):283-294, 1993.

[7] P. Lansky and R. Rodriguez. Two-compartment stochastic model of a neuron. Physica.D:.Nonlinear.Phenomena., 132(1-2):267-286, 1999.

[8] M. J. Chacron, K. Pakdaman, and A. Longtin. Interspike interval correlations, memory, adaptation, and refractoriness in a leaky integrate-andfire model with threshold fatigue. Neural Computation, 15(2):253-278, 2003.

[9] O. Avila-Akerberg and M. J. Chacron. Nonrenewal spike train statistics: causes and functional consequences on neural coding. Experimental Brain Research, 210(3-4):353-371, 2011.

[10] A. K. Vidybida and K. G. Kravchuk. Delayed feedback makes neuronal firing statistics non-markovian. Ukrainian Mathematical Journal, 64(12):1587-1609, 2012.

[11] H. L. Bryant and J. P. Segundo. Spike initiation by transmembrane current: a white-noise analysis. The Journal of Physiology, 260(2):279314, 1976.

[12] Z. F. Mainen and T. J. Sejnowski. Reliability of spike timing in neocortical neurons. Science, 268(5216):1503 -1506, 1995.

[13] R. B. Stein. Some models of neuronal variability. Biophysical.Journal, 7(1):37-68, 1967.

[14] A. N. Burkitt. A review of the integrate-and-fire neuron model: I. homogeneous synaptic input. Biological Cybernetics, 95(1):1-19, 2006.

[15] J. P. Segundo, D. Perkel, H. Wyman, H. Hegstad, and G. P. Moore. Input-output relations in computer-simulated nerve cell. Kybernetic, 4(5):157-171, 1968.

[16] R. Jolivet, T. J. Lewis, and W. Gerstner. Generalized integrate-and-fire models of neuronal activity approximate spike trains of a detailed model to a high degree of accuracy. Journal of Neurophysiology, 92(2):959-976, 2004 .

[17] R. Jolivet, A. Rauch, H. Lüscher, and W. Gerstner. Predicting spike timing of neocortical pyramidal neurons by simple threshold models. $J$ Comput Neurosci, 21(1):35-49, 2006. 
[18] O. Vidybida. Output stream of a binding neuron. Ukrainian Mathematical Journal, 59(12):1819-1839, 2007.

[19] O. K. Vidybida. Output stream of leaky integrate and fire neuron. Reports of the National Academy of Science of Ukraine, 2014(12):18-23, 2014.

[20] R. A. Nicoll and C. E. Jahr. Self-excitation of olfactory bulb neurones. Nature, 296(5856):441-444, 1982.

[21] J. M. Bekkers. Neurophysiology: Are autapses prodigal synapses? Current Biology, 8(2):R52-R55, 1998.

[22] J. L. Doob. Stochastic processes. Wiley, 1953.

[23] A. K. Vidybida. Output stream of binding neuron with delayed feedback. In J. Józefczyk, W. Thomas, and M. Turowska, editors, 14th International Congress of Cybernetics and Systems of WOSC, Wroclaw, Poland, September 9-12, 2008, pages 292-302. Oficyna Wydawnicza Politechniki Wroclawskiej, 2008.

[24] E. M. Izhikevich. Simple model of spiking neurons. IEEE Transactions on Neural Networks, 14(6):1569-1572, 2003.

[25] R. Kobayashi, Y. Tsubo, and S. Shinomoto. Made-to-order spiking neuron model equipped with a multi-timescale adaptive threshold. Frontiers in Computational Neuroscience, 3(9):1-11, 2009. 